\title{
Investigation on the indentation behavior of sandwich beams using crushable and hyperelastic foam cores
}

\author{
J. Amorim ${ }^{1}$ and G. Pitarresi ${ }^{2, a}$ \\ ${ }^{1}$ Centro Ricerche Fiat, Structural Assessment \& Optimization, Orbassano (TO), Italy. \\ ${ }^{2}$ University of Palermo, Dipartimento di Meccanica, Palermo, Italy
}

\begin{abstract}
In this work the indentation behaviour of sandwich beams is studied adopting the classical approach representing the core material as a Winkler-type foundation. It is shown how most of the proposed theories can be derived from a general fourth order linear differential equation expressing the equilibrium of the indented beam skin. Different assumptions on the supporting boundary constraints and materials constitutive behaviour, lead to simplifications of the general equation and to the prediction of different indentation features. An extension of the Segment-Wise approach recently proposed in the literature is in particular presented with potential to better simulate foam cores with markedly non-linear compressive behavior. Results from experimental indentation tests performed on sandwiches employing thermoplastic based matrices and foams are also presented to supplement the theoretical analysis.
\end{abstract}

\section{Introduction}

Sandwich structures employing lightweight composite skin faces and honeycomb or polymer foam cores are well established structural solutions exhibiting top performances in terms of specific flexural stiffness [1]. A main drawback is though represented by the relatively soft core materials employed, usually prone to develop permanent damage under localised loading. Typical structural sandwich applications such as panel flooring, body in white or monocoque transport structures can rather frequently experience localised static or low velocity impact loadings, which may induce permanent indentation damages due to local plastic deformation of the core. This inconvenience is usually significant since the induced damage can seriously deteriorate the residual load bearing capabilities of a sandwich structure [2-4], but can also determine other concerns in terms of aesthetic and functionality, in particular when secondary structural applications are considered.

Classical basic theories modelling the global flexural and shear rigidity in sandwich panels usually consider the core as transversely incompressible, hence lacking any capability for designing against local loading effects [1]. Different and more sophisticated analytical approaches have been proposed by many researchers, all trying to address a similar class of problems summarised as follow: a) determine the indentation law, i.e. the load versus displacement indentation curve, possibly including both the elastic and plastic range of core compressive behaviour; b) determine the critical applied load at core yielding onset; c) determine the level of permanent damages induced

\footnotetext{
a e-mail : pitarresi@dima.unipa.it
}

This is an Open Access article distributed under the terms of the Creative Commons Attribution-Noncommercial License 3.0, which permits unrestricted use, distribution, and reproduction in any noncommercial medium, provided the original work is properly cited. 
such as: the extent of residual dent, the extension of the core plastic zone, the load value at which local skin bending failure occurs.

The most popular approach to the study of the indentation behaviour in sandwiches with transversely compressible cores consists in solving the equilibrium equations of the loaded skin face represented as a beam or plate resting on a Winkler type foundation [2]. Solutions limited to an elastic compression behaviour of the foundation have been provided by many authors [2,3,5-9]. Solutions are available for both sandwich beams $[2,3,5,10,11]$ and plates $[6-9,11]$. In $[6,7,9]$ a two parameters elastic foundation approach is used able to include also shear forces at the face-core interface while a justification for neglecting them in the case of sandwich beams, thus considering only normal forces (assumption found in the in the majority of works) is provided in [10]. Solutions are most frequently provided for the fully backed sandwich where the unloaded face is resting against a rigid foundation [2,3,5,9,11-14]. Solutions for edge supported beams (e.g. three point bending configuration in $2 \mathrm{D}$ ) are provided by superposition approaches, e.g. by simply adding the indentation displacement contribution to the mid span bending and shear displacements measured with the classic approach [15]. A more rigorous approach is proposed by Steeves at al [10] in which a solution for an elastic-perfectly-plastic foundation constitutive behaviour is also derived. In [6-8] solutions for edge supported and edge clamped panels with the core behaving as an elastic foundation are also provided, and the influence of a distributed load, as opposed to the point load assumption, is discussed in [8]. The indentation behaviour beyond the elastic limit of the foundation has been generally treated by representing the yielding behaviour of the foam as perfectly-plastic ("Crushable" foam) and solutions are provided in [2-3,10-13,15] based on different solutions to the differential equations problem. Solutions for the important estimation of residual dents after core yielding are more rare to find $[3,4]$, while the segment-wise approach proposed in [14] has good but yet unexplored potentials for dealing with the evaluation of residual dents in foamed core sandwiches. Recently the sandwich panels higher order theory approach [16] has also been used to derive the indentation law in the core elastic compression range for both fully backed and edge supported beams, with interesting results as compared to those of classic approaches [17].

In this study analytical models for the point loaded sandwich beam indentation problem are reviewed by presenting a unified approach based on the segment-wise model recently proposed by Minakuchi et al. [14]. It is shown in particular that solutions based on elastic and elastic-perfectlyplastic constitutive laws can be derived as particular cases of this approach. The potentials of the segment-wise method are also highlighted in terms of modelling core materials with highly non linear compressive behaviours. Some preliminary experimental results are finally presented based on quasi-static indentation tests performed on sandwich beams manufactured adopting FRP faces made of thermoplastic matrices and thermoplastic foam cores. The indentation curves from the tests are compared with analytical solutions derived under different simplifying assumptions.

\section{Analytical models}

The most comprehensive approach to local indentation in sandwich consider the equilibrium differential equation of a beam (2D case) or a plate (3D case) perfectly attached to a compressible foundation. The simpler case of a sandwich beam is in particular considered. The beam skin is subject to a vertical point load on the upper face and to the foundation reactions on the lower face. Three main assumptions are in particular made which result in a simplified equilibrium equation:

a) the beam material is linear elastic and brittle (i.e. local brittle bending failure occurs without onset of plastic yielding);

b) the core material exhibits in general an elastic-perfectly plastic compressive behaviour;

c) only normal stress components in the core transverse direction are considered at the skin-core interface (requiring only one stiffness parameter to model the foundation elastic response).

Steeves et al. [10] have in particular pointed out how the above assumptions are well met by brittle FRP skins and polymer foam cores, although not limited to these material combinations. 


\subsection{Elastic-plastic foundation models}

Different solutions have been proposed under different supporting boundary conditions and material behaviour assumptions (see table 1). The main cases proposed regard:

- Point loaded sandwich beams where the lower skin is resting on a rigid surface, i.e. a fully backed sandwich beam (FB) [2,3,5,11-14];

- Point loaded and edge supported sandwich beams, i.e. three point bending scheme (TPB) [10];

- Core material behaving as a rigid perfectly plastic foundation (RPP) [10,12];

- Core material behaving as a pure linear elastic foundation (E) $[2,3,5,13,14]$;

- Core material behaving as an elastic-perfectly plastic foundation (EPP) $[2,3,10,11,15]$.

The general expression for the equilibrium equation of the beam is a fourth order linear differential equation $[2,3,14]$, whose coefficients are functions of the core and skin geometric, elastic and constitutive properties. This equation can be written as:

$$
l \cdot \frac{d^{4} w(x)}{d x^{4}}+m \cdot \frac{d^{2} w(x)}{d x^{2}}+k \cdot w(x)=c
$$

where $x$ is the beam axis coordinate centred on the loading point and $w$ the transverse deflection orthogonal to the beam axis. The presence of coefficients $l, k, c$ depends on the particular core constitutive behaviour, while coefficient $m$ appears only in the case of a TPB loading configuration (see table 1 for which coefficients are present in the various models, and table 2 for their definitions).

Table 1. Static indentation features in sandwich beams predicted by different analytical approaches.

\begin{tabular}{|l|c|c|c|c|c|c|c|}
\hline \multirow{2}{*}{ Contributions } & \multirow{2}{*}{$\begin{array}{c}\text { Type of } \\
\text { support }\end{array}$} & \multicolumn{3}{|c|}{ Mid-span displ. vs Load curve } & $\begin{array}{c}\text { Load at } \\
\text { core yield } \\
\text { setting }\end{array}$ & $\begin{array}{c}\text { Load at skin } \\
\text { bending } \\
\text { failure }\end{array}$ \\
\hline Soden [12] & $F B$ & $\times$ & - & - & - & - & $\times$ \\
\hline Shuaeib et al. [13] & $F B$ & - & $\times$ & - & - & $\times$ & $\times$ \\
\hline$[2,3]$ & $F B$ & $\times$ & $l, k$ & $l, c$ & - & $\times$ & - \\
\hline Steeves et al. [10] & $T P B$ & $\times$ & $l, m, k$ & $l, m, c$ & - & $\times$ & - \\
\hline Minakuchi et al. [14] & $F B$ & $\times$ & $\times$ & $\times$ & $l, k, c$ & - & - \\
\hline
\end{tabular}

Table 2. Definition of eq. (1) coefficients.

\begin{tabular}{|c|c|c|}
\hline Coeff. & Definition & notes \\
\hline$l$ & $E_{f} I_{f}$ & $\begin{array}{c}\text { Flexural rigidity of the beam ( } E_{f} \text { longitudinal Young's modulus of beam, } I_{f} \\
\text { inertia moment); }\end{array}$ \\
\hline$m$ & $P L / 4\left(t_{c}+t_{s}\right)$ & $\begin{array}{c}\text { Membrane axial force on the beam skin ( } P \text { applied point load, } L \text { TPB span } \\
\text { length, } t_{c} \text { core thickness, } t_{s} \text { skin thickness) [10] }\end{array}$ \\
\hline$k$ & $E_{c} b / t_{c}$ & $\begin{array}{l}\text { Foundation Elastic modulus ( } E_{c} \text { Core compressive stiffness, b sandwich } \\
\text { beam width); }\end{array}$ \\
\hline \multirow{2}{*}{$c$} & $-q_{i}$ & Constant stress applied to the beam (see fig. 1) \\
\hline & $-b \sigma_{c}$ & Core yield stress (value of plastic plateau in EPP behaviour). \\
\hline
\end{tabular}

Minakuchi et al. [14] have proposed a segment-wise discretisation of the core constitutive behaviour (abbreviated $S W$ in table 1) to represent more complex uniaxial compression behaviours. 
The model is proposed for a FB support type, and the beam axis is discretised into segments each responding to a portion of the material segmented behaviour. Each beam segment has a different form of equation (1) with different values of the $l, k, c$ coefficients according to the corresponding material behaviour.

\subsection{Segment-wise approach}

A segment-wise approach has recently been proposed by Minakuchi et al. [14] in order to study the indentation behaviour on core materials following complex uniaxial compression behaviours, which may include also unloading steps for residual dent evaluation. In particular the approach proposed in [14] is fully developed and implemented on a periodic shape aluminium honeycomb core. It is shown here that the segment-wise approach can be applied to model a foam core with a generic nonlinear monotonically increasing compression curve (see fig. 1a). The set of lines discretising the non linear compression curve in fig. 1 can be considered as a succession of linear equations:

$$
\sigma(w(x))=k_{i} w(x)+q_{i} \quad \text { where } \delta_{\mathrm{i}}<w(x)<\delta_{\mathrm{i}+1} \text { and } n \text { number of segments used }
$$

where $\delta_{i}$, is the unknown position along the beam axis at which the material changes his compressive behaviour from $k_{i}, q_{i}$ (outer part) to $k_{i+1}, q_{i+1}$ (inner part).

Applying the SW approach results in a system of $n$ linear differential equations:

$$
E_{f} I_{f} \frac{d^{4} w(x)}{d x^{4}}+k_{i} \cdot w(x)+q_{i}=0
$$

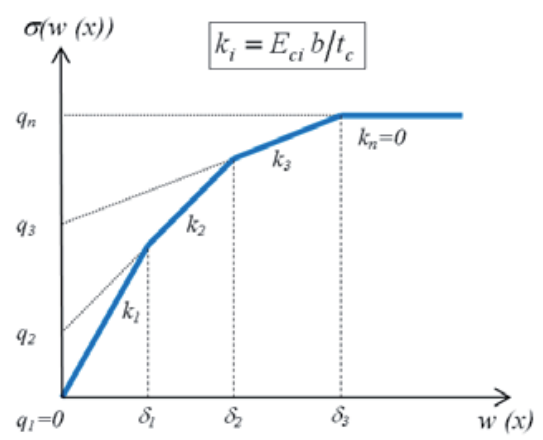

(a)

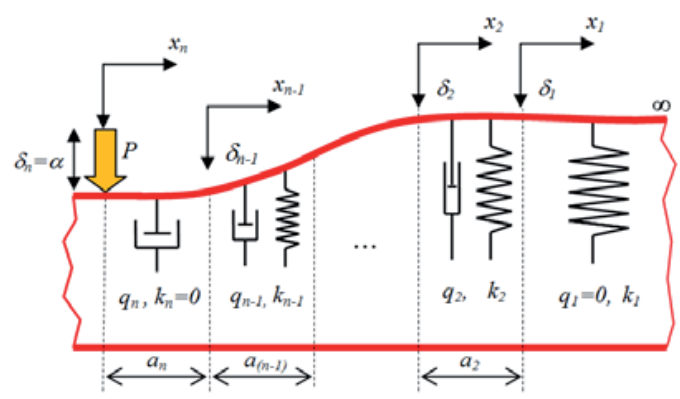

(b)

Fig. 1. a) Segmentation of a non-linear stress $v s$ displacement core compression test; b) schematic representation of a fully backed point loaded half beam, with indication of zones with material behaviour defined by $k$ and $q$ values in fig 1 a.

Minakuchi et al [14] report the general solutions for eq. (1). If for simplicity the compressive curve is considered monotonically increasing toward a horizontal plateau (as depicted in fig. 1a), only three general solutions are needed: a) for $k_{i}>0$ and $q_{i}=0$ (e.g. when $i=1$ ), b) for $k_{i}>0$ and $q_{i} \neq 0, \mathrm{c}$ ) for $k_{i}=0$ and $q_{i} \neq 0$ (e.g. when $i=n$ ). The general solutions are:

$$
\begin{aligned}
& \text { a) } w(x)=e^{-\lambda x}\left[A_{1} \sin (\lambda x)+B_{1} \cos (\lambda x)\right]+e^{\lambda x}\left[C_{1} \sin (\lambda x)+C_{1} \cos (\lambda x)\right] \\
& \text { b) } \left.w(x)=-\frac{q_{i}}{k_{i}}+e^{-\lambda x}\left[A_{1} \sin (\lambda x)+B_{1} \cos (\lambda x)\right]+e^{\lambda x}\left[C_{1} \sin (\lambda x)+C_{1} \cos (\lambda x)\right]\right\} \lambda=\sqrt[4]{\frac{k_{i}}{4 E_{f} I_{f}}} \\
& \text { c) } w(x)=-\frac{q_{n}}{24 E_{f} I_{f}} x^{4}+A_{n} x^{3}+B_{n} x^{2}+C_{n} x+D_{n}
\end{aligned}
$$


The problem has assigned a number of $5 \times n$ total boundary conditions, B.C., summarised in fig. 2 . In particular there are always 2 B.C. requiring that the solution remains bounded at the outermost end $[2,3,14]$ :

$$
\lim _{x_{1} \rightarrow \infty} w_{1}\left(x_{1}\right)=0 ; \quad \lim _{x_{1} \rightarrow \infty} w_{1}^{\prime}\left(x_{1}\right)=0
$$

Three further B.C. apply at the loading point:

$$
w_{n}(0)=\alpha ; \quad w_{n}^{\prime}(0)=0 ; \quad w_{n}^{\prime \prime \prime}(0)=\frac{P}{2 E_{f} I_{f}}
$$

in which $\alpha$ and $P$ are unknown. Finally a total $5 \times(n-1)$ B.C. apply from imposing continuity of the displacements, slopes, bending moments and shear forces at the interface between adjacent beam segments, and knowing the core displacement at which a new set of material parameters $k_{i}$ and $q_{i}$ is set:

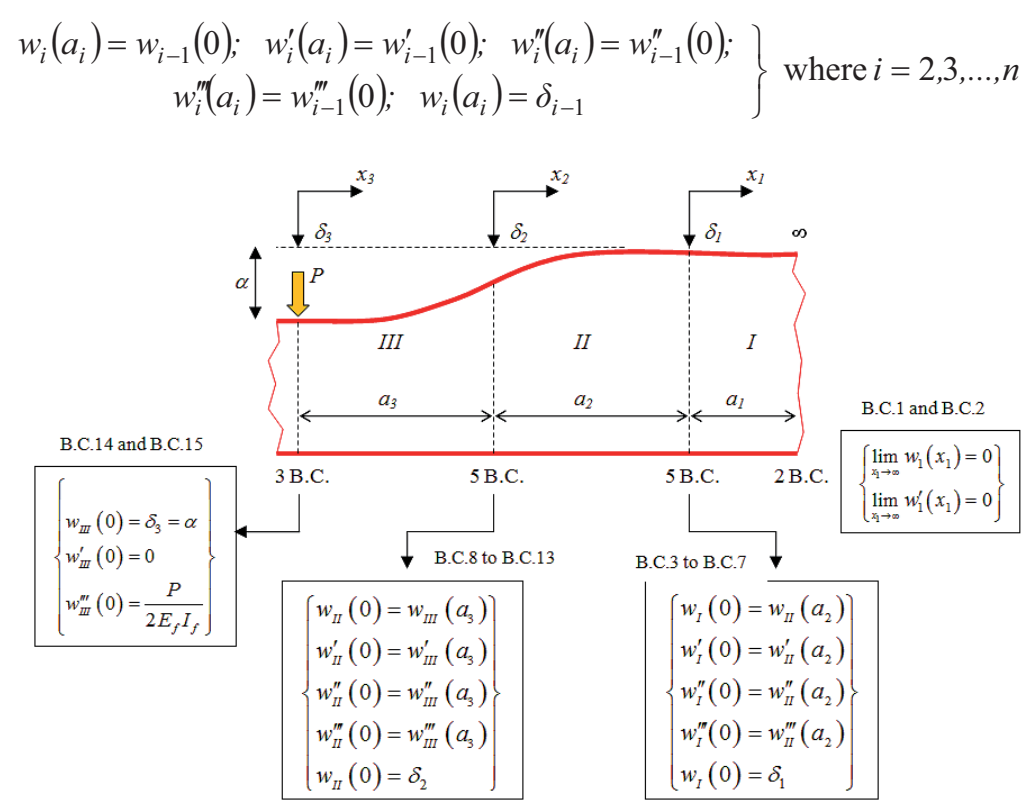

Fig. 2. Boundary conditions applicable when a three segments discretisation is chosen.

Imposing the boundary conditions to the $n$ solutions (eqs. 4) yields a system of $5 \times n$ non linear equations with $(5 \times n+1)$ unknowns: $4 \times n$ consisting in $\mathrm{A}_{i}, B_{i}, C_{i}$ and $D_{i}$ (with $i=1$ to $\left.n\right),(n-1)$ values of $a_{i}$ (with $i=2$ to $n$ ), and finally the last two unknowns consisting in $P$ and $\alpha$ (from which the indentation curve is obtained). The system is reduced to 15 equations in 15 unknowns by assigning a value to $a_{2}$ and deriving a solution. Iterating the solution for a range of chosen $a_{2}$ values yields the entire curve $P, \alpha$.

The generic final system of simultaneous $5 \times n$ equations from the application of all B.C. is nonlinear and hence not straight forward to solve. In [14] the previous model is applied to a honeycomb core structure. In this case the compressive and stretching behaviour is the same for each cell and the segmentation of the sandwich beam is easily established by dimensioning all values of $a_{i}(i=2$ to $n)$ with the same size which is the size of the elementary honeycomb cell. This allows a reduction of unknowns and a more straight forward solution of the problem. In foam cores no such information is provided for $a_{i}$. The segment-wise approach lends itself well as a unifying approach to the study of 
sandwich indentation, including different assumptions all based on modelling the core as a compressible foundation. In fact the basic cases of elastic (E) and elastic perfectly plastic (EPP) foundations are immediately derived from the above SW formulation according with the following opportune choice of the material compression curve segmentation:

- E-foundation: only one linear elastic segment is considered with the elastic foundation coefficient expressed as in $[2,3,5]$. In this case there will be only 5 B.C. (3 at the loading point, with $w(0)=B_{l}$, and 2 at the outermost beam end as in fig. 2) and 5 unknowns $\left(A_{l}, B_{l}, C_{l}, D_{l}\right.$ and $P$, with $\left.B_{1}=\alpha\right)$;

- EPP-foundation: The material behaviour is approximated with only two segments, one linear elastic and the successive one perfectly horizontal, representing the plastic plateau. In this case there will be 10 B.C. ( 3 at the loading point, and 2 at the outermost beam end, and 5 at the interface between zones $a_{1}$ and $a_{2}$, i.e. the transition between the elastic and plastic core) and 10 unknowns ( $P, \alpha, A_{i}, B_{i}, C_{i}, D_{i}$ with $\left.\mathrm{i}=1,2\right)$. After assigning values to $a_{2}$, a subsystem of linear simultaneous equation is obtained with only three unknowns: $A_{1}, B_{2}$ and $P$. The final solution in terms of $P$ and $\alpha$ is then straightforward.

If a non-linear core compressive behaviour is obtained and a SW approach used with a number of segments higher than 2 , it is found that the final solving system is always non-linear.

\section{Experimental results}

In this work three sandwich beams have been assembled and tested under quasi-static indentation loading with the purpose to compare the measured indentation curves with those predicted using the EPP approach commented in section 2. A summary of the materials employed is reported in table 3, together with the values of physical parameters used in the analysis.

Table 3. Sandwich beams data. Properties with the $M$ superscript have been measured (uncertainty equal to standard deviation from 5 tested samples), and superscript $S$ is used for data provided by the supplier.

Materials employed: I Tepex ${ }^{\circledR}$ dynalite 102-RGUD385 (60\% vol. fibre content); II manufactured by hand lay-up (45\% vol. fibre content); ${ }^{\text {III }}$ Tepex ${ }^{\circledR}$ dynalite $102-R G 600$ (45\% vol. fibre content); ${ }^{\text {IV }}$ Zotek ${ }^{\circledR}$ N B50 polyamide $50 \mathrm{~kg} / \mathrm{m}^{3}$; ${ }^{V}$ Propozote ${ }^{\circledR}$ PPA30 polypropilene $30 \mathrm{~kg} / \mathrm{m}^{3}$; ${ }^{\mathrm{VI}}$ Herex ${ }^{\circledR} \mathrm{C} 70-55\left(60 \mathrm{~kg} / \mathrm{m}^{3}\right)$.

\begin{tabular}{|c|c|c|c|c|c|c|c|c|c|}
\hline \multicolumn{3}{|c|}{ Beam specimen } & \multicolumn{3}{|c|}{ Skin } & \multicolumn{4}{|c|}{ Core } \\
\hline$I D$ & $\begin{array}{c}b \\
{[\mathrm{~mm}]}\end{array}$ & $\begin{array}{c}L \\
{[\mathrm{~mm}]}\end{array}$ & laminate & $\underline{\underline{\mathrm{t}_{\mathrm{s}}}}$ & $E_{f}[\mathrm{GPa}]$ & foam & $\underline{\mathrm{t}_{c}}[\mathrm{~mm}]$ & $\begin{array}{c}E_{c} \\
{[M P a]}\end{array}$ & $\begin{array}{c}\sigma_{\mathrm{c}} \\
{[\mathrm{Mpa}]}\end{array}$ \\
\hline $\mathrm{S} 1$ & 25 & 245 & $\begin{array}{c}\text { Glass/PA6 }{ }^{\mathrm{I}} \\
{\left[0^{\circ}\right]_{\text {unid }}} \\
\end{array}$ & 2 & $46 \pm 1.5^{M}$ & $\mathrm{PA}^{\mathrm{IV}}$ & 30 & $1.5^{M}$ & $0.14^{M}$ \\
\hline $\mathrm{S} 2$ & 40 & 150 & $\begin{array}{c}\text { Glass/epoxy }{ }^{\text {II }} \\
{\left[0^{\circ} / 90^{\circ}\right]_{\mathrm{s}}}\end{array}$ & 1 & $18.1 \pm 1.1^{M}$ & $\mathrm{PP}^{\mathrm{V}}$ & 20 & $1.22^{M}$ & $0.11^{M}$ \\
\hline S3 & 20 & 119 & $\begin{array}{l}\text { Glass/PA6 } \\
{\left[0^{\circ} / 90^{\circ}\right]_{\text {st }}{ }^{\text {III }}}\end{array}$ & 0.5 & $20.2 \pm 0.6^{M}$ & $\mathrm{PVC}^{\mathrm{VI}}$ & 5 & $58^{S}$ & $0.85^{S}$ \\
\hline
\end{tabular}

All indentation curves were measured on a Instron 3367 testing rig equipped with a $1 \mathrm{kN}$ load cell. A cylindrical indenter with diameter of $25 \mathrm{~mm}$ was used. Figure 3 shows pictures of samples S1 and $\mathrm{S} 3$ during the indentation test.

The main purpose in the experimental analysis presented in this work was the assessment of the RPP [12] and EPP analytical approaches on sandwich concepts employing thermoplastic matrices or foams with a markedly non linear behaviour. Future work is under way to apply a SW approach with a higher number of segments to better model the material behaviour in the case of marked nonlinearity. 

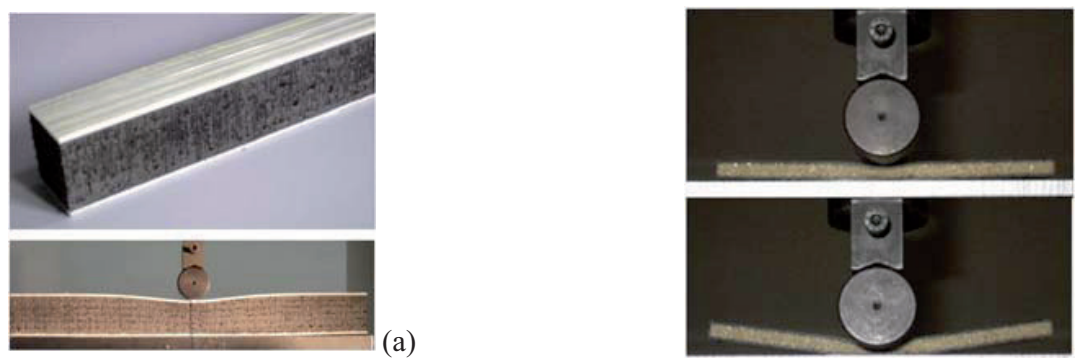

(b)

Fig. 3. Sample S1 (a) and S3 (b) during the indentation test (cylindrical indenter diameter $25 \mathrm{~mm}$ ).

Figure 4 reports data from a flatwise compression test on a polypropylene foam. It is observed that the behaviour is highly non linear. The material is also able to recover from high strain deformations, resembling a hyperelastic behaviour. Figure 4a shows a residual $20 \%$ compression after a $60 \%$ total compression. This residual compression is largely further recovered after a certain relaxation time although some permanent damage was observed from successive loading cycles. In Fig. $4 \mathrm{~b}$ the compression curve up to about $50 \%$ strain (before marked densification occurs) is considered and an elastic-perfectly-plastic curve built according with the following criteria: the elastic slope is equal to the slope of the initial part of the foam compression curve; the value of the plateau is chosen such to have the area under the EPP curve equal to the area under the non-linear measured curve. The parameters of the approximated EPP compression curve were than used in the analytical prediction of the indentation curve (results shown in fig. $5 b$ ). The same procedure is also followed to derive the EPP parameters in the case of sample 1.
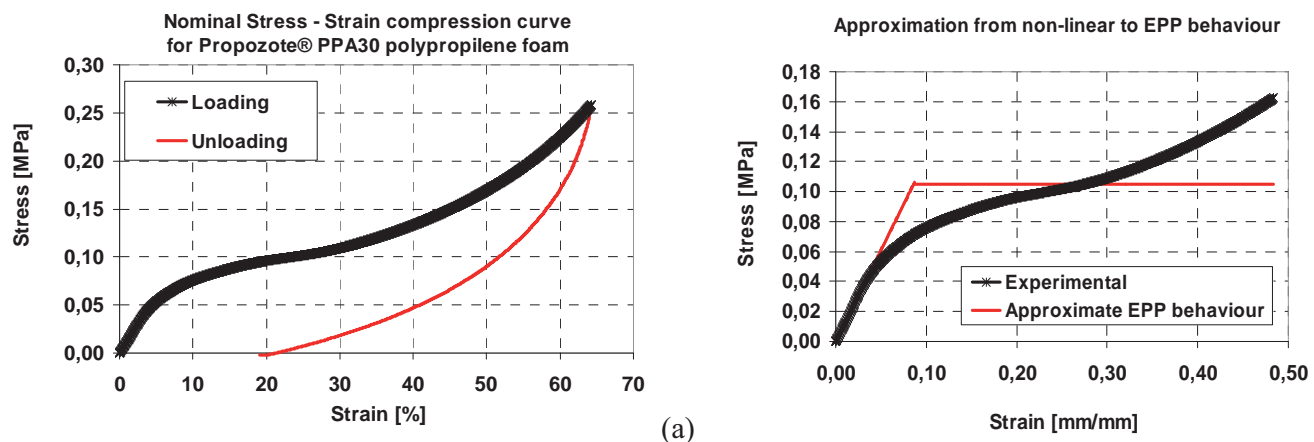

(b)

Fig. 4. Results from flatwise compression test on PPA30 foam performed according to ASTM C365.

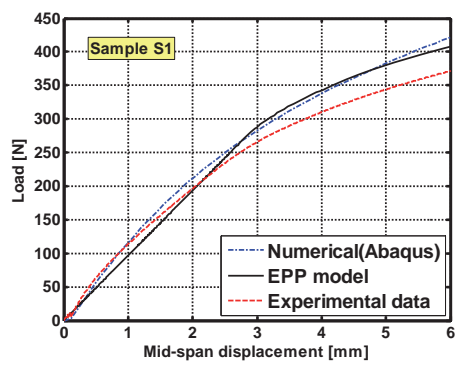

(a)

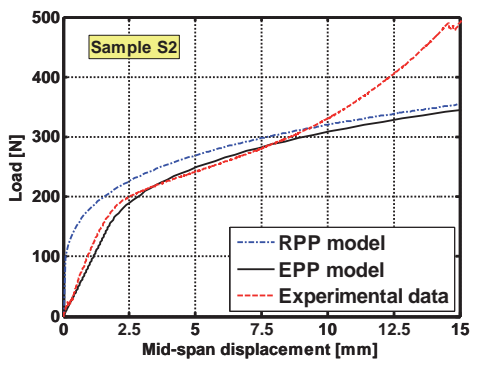

(b)

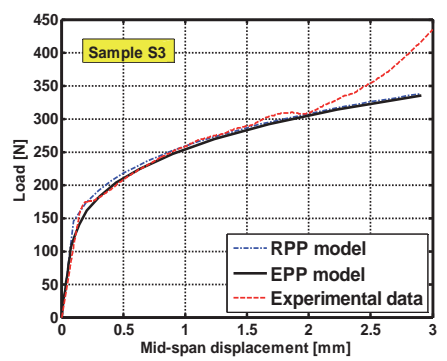

(c)

Fig. 5. Indentation curves: comparison of numerical, analytical and experimental results. 
Figure 5 shows a comparison of analytical and experimental indentation curves for tested samples. It is found that the best match is for sample 3 where a crushable PVC foam is used. The adoption of a thermoplastic reinforced laminate in the skin face does not affect the effectiveness of the EPP approach in this case [3]. Also the RPP approach performs well for this case since the elastic stiffness of the foam is high, and the thin foam thickness does not allow to accumulate significant elastic compression before yielding. In the case of sample 2 the limits of the RPP prediction are more evident due to the high elastic deformation of the core. In the case of sample 1 a numerical prediction was also obtained which matches well with the analytical prediction. The foam behaviour in the numerical model was simulated implementing the hyperelastic constitutive models proposed by Abaqus, and based on the Ogden strain energy formulation. The Abaqus Hyperfoam strain energy potential is used, whose constitutive parameters have been determined from a lest-square fitting procedure which uses the experimental foam uniaxial compression test data.

\section{Conclusions}

In this work a unified approach summarised by eq. 1 is presented to describe the indentation behaviour of both fully backed and edge supported sandwich beams under local loading. The indentation problem is in particular modelled by studying the penetration of an elastic beam (sandwich skin face) on a compressible foundation (sandwich core). The work shows also that the foam core material constitutive compression behavior can be generalised by adapting a segmentwise (SW) model proposed in [14]. Simpler constitutive behaviours such as pure elastic and elasticperfectly-plastic (EPP) are in this case obtained as special cases according with the number and features of segments chosen in the SW approach. It is also evidenced the potential of the SW approach to analyse highly non linear foam core compression behaviours. Finally some experimental data are presented from testing of thermoplastic sandwich beams. A comparison is made between the experimental data and the analytical predictions obtained from employing an EPP approximation to simulate the non-linear compression behaviour of thermoplastic foam cores.

\section{Acknowledgements}

The authors wish to acknowledge the European Union for supporting the work through a Marie Curie fellowship and the support from all members of the Marie Curie Research Training Network MOMENTUM.

\section{References}

1. F.J. Plantema, Sandwich construction (New York, Wiley, 1966)

2. S. Abrate, Appl Mech Rev 50-2, 69 (1997)

3. D. Zenkert, A. Shipsha, K. Person, Composites: Part B, 33, 511 (2004)

4. V. Koissin, A. Shipsha, Compos Struct, 68, 57 (2008)

5. J.L. Abot, I.M. Daniel, E.E. Gdoutos, J Sandw Struct Mater, 4-2, 157 (2002)

6. O.T. Thomsen, Compos Struct, 25, 511 (1993)

7. O.T. Thomsen, Compos Struct, 30, 85 (1995)

8. S.M. Lee, T.K. Tsotsis, Compos Sci Technol, 60, 1147 (2000)

9. M. Yang, P. Qiao, Compos Sci Technol, 65, 551 (2005)

10. C. Steeves, N. Fleck, International Journal of Mechanical Sciences, 46, 561 (2004)

11. V. Koissin, A. Shipsha, V. Rizov, Compos Struct, 64, 129 (2004)

12. P.D. Soden, J Strain Anal 31, 353 (1996)

13. F.M. Shuaeib, P.D. Soden, Compos Sci Technol 57, 1249 (1997)

14. S. Minakuchi, Y. Okabe, N. Takeda, Compos Part A, 39, 133 (2008)

15. M. Sadighi and H. Pouriayevali, J Sandw Struct Mater, 10-6, 499 (2008)

16. Y. Frostig, Composites: Part B, 34, 83 (2003)

17. M. Saadati, M. Sadighi, Proc. IMechE Part G: J. Aerospace Engineering, 223, 825 (2009) 